\title{
Reducing overtreatment of prostate cancer by radical prostatectomy in Eastern Ontario: a population-based cohort study
}

\author{
Luke Witherspoon MD MSc, Johnathan L. Lau BSc, Rodney H. Breau MD MSc, \\ Christopher Knee ND MSc, Michael Fung Kee Fung MD, Robin Morash RN, Ranjeeta Mallick PhD, \\ Ilias Cagiannos MD, Christopher Morash MD, Luke T. Lavallée MD MSc
}

\section{Abstract}

Background: Canadian guidelines recommend against population-based screening for prostate cancer because of the risk of overdiagnosis and overtreatment. We sought to assess whether a higher proportion of patients receiving surgery had clinically significant cancer over time.

Methods: All hospitals in Eastern Ontario that perform prostatectomy participate in a Prostate Cancer Community of Practice, which prospectively maintains a database for the region. Using these data, we conducted a retrospective cohort study that included all patients who underwent prostatectomy from 2009 to 2015 in the region. We examined trends in biopsy findings, clinical stage, prostate-specific antigen level and Gleason score. We then determined whether the proportion of patients with clinically significant cancer (Gleason score $\geq 7$ or stage pT3) increased over time.

Results: During the study period, 1897 patients underwent prostatectomy in Eastern Ontario (mean 271 surgeries/yr). The proportion of patients who were determined to have National Comprehensive Cancer Network intermediate or high-risk disease increased from $46.7 \%$ in 2009 to $90.2 \%$ in 2015 . The proportion of men with clinically significant cancer on prostatectomy increased from $59.7 \%$ in 2009 to $93.1 \%$ in 2015 . Adjusted analyses suggested that the proportion of patients with clinically significant cancer increased by $5 \%$ per year during the study period.

Interpretation: There has been a change in the tumour characteristics of patients who undergo prostatectomy in Eastern Ontario. In recent years, almost all patients have had clinically significant cancer, which suggests that overtreatment of prostate cancer has decreased.

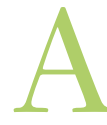

bout $15 \%$ of men in Canada receive a diagnosis of prostate cancer in their lifetime. ${ }^{1}$ Overtreatment of prostate cancer is a public health concern because it exposes men to risks that may not be necessary. ${ }^{2}$ Historically, $50 \%-75 \%$ of patients with low-risk cancer received radical treatment, such as surgery or radiotherapy. ${ }^{3}$ Improved understanding of the natural history of prostate cancer has made active surveillance the preferred initial treatment for patients with low-risk disease..$^{3-7}$

We have previously shown that active surveillance is the most common initial approach for patients with low-risk disease at The Ottawa Hospital. ${ }^{4}$ Contrary to low-risk cancers, studies show that men with intermediate and high-risk disease are at considerable risk of metastasis and cancerspecific death, and treatment is advocated for patients with a life expectancy of more than 10 years. ${ }^{8,9}$ We sought to assess whether urologists in Eastern Ontario have increased the threshold of risk for recommending prostatectomy to patients in an effort to reduce overtreatment. We hypothesized that an increasing proportion of men receiving prostatectomy in our population would have clinically significant cancer.

\section{Competing interests: None declared.}

This article has been peer reviewed.

Correspondence to: Luke Lavallée, lulavallee@ toh.ca

CMAJ Open 2018. DOI:10.9778/cmajo.20170149 


\section{Methods}

\section{Study design and data sources}

All patients who underwent radical prostatectomy in Eastern Ontario (population $~ 1.2$ million) between Jan. 1, 2009, and Dec. 31, 2015, were included in the cohort. In Eastern Ontario, prostatectomy procedures are routinely done at 3 hospitals: The Ottawa Hospital, Montfort Hospital and the Queensway-Carleton Hospital. These hospitals participate in a Prostate Cancer Community of Practice. The community of practice consists of surgeons, radiation oncologists, medical oncologists, pathologists, radiologists, specialized nurses and administrators involved with prostate cancer care in our region. The Prostate Cancer Community of Practice database is prospectively maintained and collects data for every patient who undergoes radical prostatectomy in these 3 hospitals. The database is stored and managed at The Ottawa Hospital.

\section{Study population}

Patients who underwent open, pure laparoscopic, and roboticassisted laparoscopic radical prostatectomies were included in the cohort. Patients who underwent salvage prostatectomies after radiotherapy were excluded.

We recorded patient and tumour characteristics. Patient characteristics included age, prostate-specific antigen (PSA) level before surgery and prostate cancer clinical stage based on digital rectal examination. Tumor characteristics included Gleason score on biopsy, Gleason score on prostatectomy, and pathologic stage. A review of medical records was used to complete missing data when possible. Prostate biopsies included standard template and targeted biopsies as per clinical indication. Pathologic processing of biopsies and surgical specimens followed institutional standardized protocols and were not changed during the study period.

We categorized patients into risk groups using National Comprehensive Cancer Network criteria (low-risk, stage cT2a or lower, PSA < $10 \mathrm{ng} / \mathrm{mL}$ or biopsy Gleason score < 6; intermediate-risk, stages cT2b-T2c, PSA $10-20 \mathrm{ng} / \mathrm{mL}$ or biopsy Gleason score 7; high-risk, stage cT3a or higher, PSA $>20 \mathrm{ng} / \mathrm{mL}$ or biopsy Gleason score $\geq 8$ ). Risk stratification was not possible for all patients owing to missing data. In addition, we categorized patients as having clinically significant or clinically insignificant cancer using pathologic variables. Clinically significant cancer was defined as prostatectomy Gleason score of 7 or greater, or pathologic stage T3 or higher, because these factors are associated with increased risk of cancerspecific death. ${ }^{9}, 10$ In addition, we identified a subcohort of patients with Gleason 6 tumours on biopsy to examine what patient and tumour characteristics may lead patients to select surgery for low-risk disease.

\section{Data analysis}

We examined trends in clinical (biopsy Gleason score, clinical stage, PSA level) and pathological characteristics (surgical Gleason score, pathologic stage) by year. We performed univariable and multivariable analyses to determine the association between patient age, year of surgery, surgical approach, and hospital with clinically significant cancer after prostatectomy using a log binomial regression. We used SAS software version 9.4 (Cary, NC) for Windows for all analyses.

\section{Ethics approval}

We obtained approval from the institutional ethics board of each hospital that contributed data to this study.

\section{Results}

During the study period, 1897 patients underwent prostatectomy in Eastern Ontario. The mean annual number of prostatectomies was 271 ( \pm standard deviation $[S D] 25)$. The annual case volume in the population peaked at 304 in 2011 and decreased in each subsequent year to 247 in 2015 . The mean age of patients was $62.3( \pm 6.5)$ years, and the mean preoperative PSA level was $8.5( \pm 9.2)$ (Table 1$)$. Of the surgeries performed, 677 prostatectomies were done using a laparoscopic (robotic-assisted or pure laparoscopic) approach, and 802 prostatectomies were done using an open approach. Most of the laparoscopic surgeries were robotic-assisted.

\section{Preoperative patient and tumour characteristics}

During the study period, the proportion of patients who underwent prostatectomy with stage cT2 and cT3 cancers increased from $15.6 \%$ to $40.7 \%$. The proportion of tumours with a biopsy Gleason score of 7 or higher increased from $38.1 \%$ to $85.1 \%$. National Comprehensive Cancer Network preoperative risk stratification was mainly possible for patients who underwent treatment at The Ottawa Hospital $(n=1456)$. The proportion of patients who underwent prostatectomy with National Comprehensive Cancer Network intermediate or high-risk disease increased from $46.7 \%$ in 2009 to $90.2 \%$ in 2015 (Figure 1A).

\section{Postoperative tumour characteristics}

During the study period, the proportion of tumours with a Gleason score of 7 or higher increased from $55.9 \%$ to $92.7 \%$, and the proportion with stage pT3 disease at prostatectomy increased from $25.2 \%$ to $45.1 \%$ (Figure $1 \mathrm{~B}$ and $1 \mathrm{C}$ ). The proportion of men with clinically significant cancer (Gleason score $\geq 7$ or stage pT3) at prostatectomy increased from $59.7 \%$ to $93.1 \%$ (Figure 1D).

Multivariable analyses showed that year of surgery, patient age and preoperative PSA level were significantly associated with clinically significant cancer at prostatectomy (Table 2). With every year between 2009 and 2015, 5\% more patients had clinically significant cancer $(95 \%$ confidence interval [CI] 4\%-6\%). Type of hospital (academic v. community) was not significantly associated with clinically significant cancer.

\section{Gleason 6 subcohort}

We identified 444 patients with tumours with a Gleason score of 6 on preoperative biopsy who underwent surgery. Of these patients, $281(63.3 \%)$ had clinically significant cancer (Gleason score $\geq 7$ or stage pT3) at prostatectomy. Of the 163 patients 


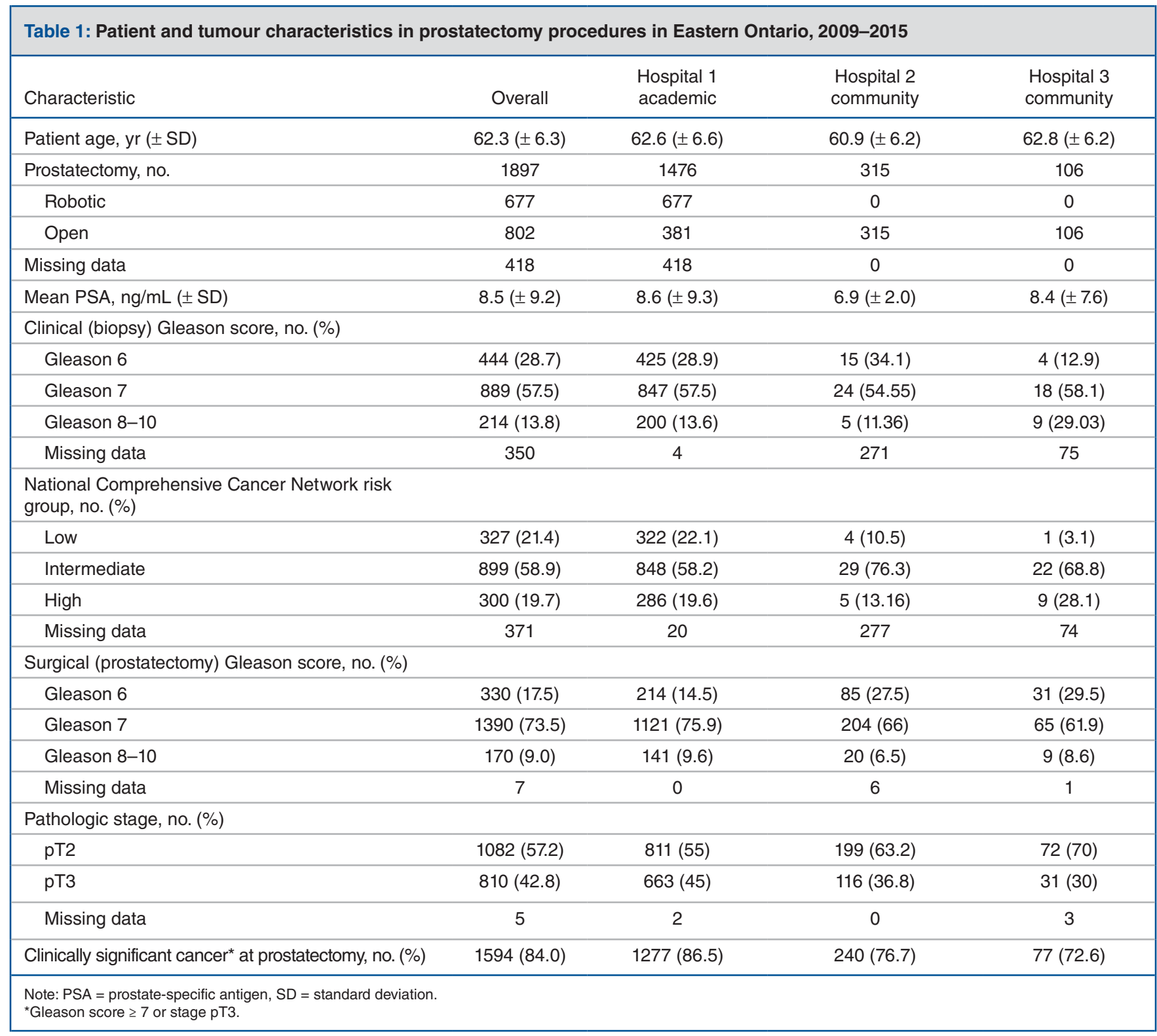

who did not have clinically significant cancer at prostatectomy, 152 had a preoperative PSA level and clinical stage available, $87(53.4 \%)$ patients were less than 60 years of age, $14(8.6 \%)$ patients had a preoperative PSA level greater than 10 , and 16 $(9.8 \%)$ patients had a clinical stage of $\mathrm{T} 2$ or higher.

\section{Interpretation}

Overtreatment was identified as a major drawback of population-based screening for prostate cancer by Canadian policy-makers in 2014. ${ }^{2}$ This legitimate concern was predicated on the high number of men with low-risk prostate cancer who received treatment (surgery or radiation) with unclear benefit but well-defined adverse effects of treatment. Our study involving patients in Eastern Ontario shows a significant increase in the proportion of patients receiving surgery who have clinically significant cancer. By 2015, 93\% of patients who received surgery had cancer characteristics (grade and stage) associated with moderate to high risk of metastases or cancer-specific death within 10 years. ${ }^{8}$ These data suggest physicians have changed the way they select men for surgery to reduce overtreatment.

Overdiagnosis and overtreatment of prostate cancer are valid concerns. Data from the European Randomized Study of Screening for Prostate Cancer (ERSPC) trial estimated a $40 \%$ rate of overdiagnosis in populations who underwent screening. ${ }^{9}$ Since large screening trials started, randomized and observational studies have improved our knowledge regarding the natural history of low-risk prostate cancer, and it is now well accepted that most low-grade cancers are associated with minimal risk of cancer-related death. ${ }^{8,10-12}$ For this reason, reducing the number of men with low-risk cancer who receive a diagnosis and subsequent treatment is important. Although eliminating population-based prostate cancer screening reduces low-risk cancer diagnoses, it disregards the 


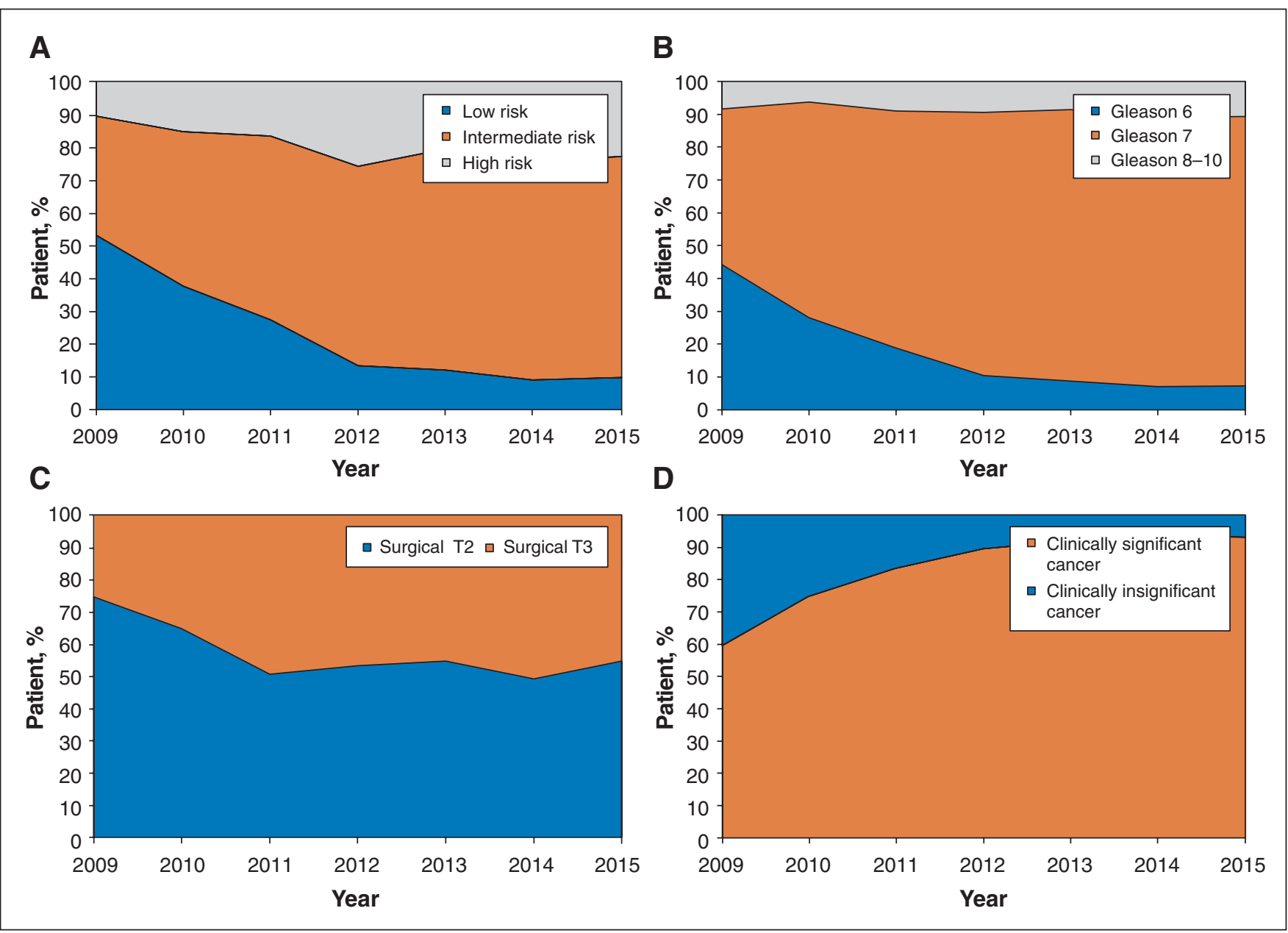

Figure 1: (A) National Comprehensive Cancer Network risk stratification for patients undergoing radical prostatectomy in Eastern Ontario between 2009 and 2015. (B) Pathologic (prostatectomy) Gleason score of patients between 2009 and 2015. (C) Pathologic (prostatectomy) T stage for patients between 2009 and 2015. (D) Proportion of patients with clinically significant cancer at prostatectomy between 2009 and 2015. Clinically significant cancer is defined as a Gleason score of 7 or higher, or stage pT3 disease.

Table 2: Association between patient and hospital characteristics and clinically significant cancer at prostatectomy

\begin{tabular}{|lcc|}
\hline Characteristic & Univariable RR $(95 \% \mathrm{Cl})$ & Multivariable RR (95\% Cl) \\
\hline Surgery year (increase by 1 yr) & $1.04(1.04-1.05)$ & $1.05(1.03-1.06)$ \\
\hline Patient age (increase by $1 \mathrm{yr})$ & $1.01(1.01-1.02)$ & $1.01(1.007-1.013)$ \\
\hline Preoperative PSA (increase by 1 unit) & $1.00(1.00-1.01)$ & $1.00(1.00-1.01)$ \\
\hline Academic v. community hospital & $1.14(1.08-1.21)$ & $1.05(0.97-1.14)$ \\
\hline Note: $\mathrm{Cl}=$ confidence interval, PSA = prostate-specific antigen, RR = relative risk. & \\
\hline
\end{tabular}

benefit of diagnosing higher risk cancers that would benefit from treatment. Men with intermediate (Gleason 7) and highrisk (Gleason 8-10) cancers have prostate cancer-specific risks of death of $40 \%-80 \%$ and $60 \%-90 \%$ by 20 years without treatment, respectively. ${ }^{8}$ To render prostate cancer screening more beneficial, physicians must better select men to receive treatment after diagnosis.

Active surveillance is now the recommended first-line treatment for most men with Gleason 6 cancer. ${ }^{12-15} \mathrm{We}$ pre- viously reported that $67 \%$ of men with Gleason 6 cancer at The Ottawa Hospital receive active surveillance, regardless of PSA or clinical stage. ${ }^{4}$ This study complements these data by showing that almost all patients who received surgery in Eastern Ontario had clinically significant (Gleason $\geq 7$ or stage pT3) disease. Furthermore, the proportion of patients with clinically significant cancer who underwent surgery has increased by $5 \%$ per year and continues to rise. This trend was seen at academic and community centres, 
and among patients who received both open and robotic assisted/laparoscopic approaches.

An alternative explanation for the trends we saw is that decreased screening in the population has led to a diagnostic stage migration, whereby only more advanced cases are being detected. Although diagnostic stage migration may be contributing to the trends seen, our previous report that suggested that most men with low-risk disease are selecting active surveillance makes it likely changes in treatment selection are the main determinant. This argument is further supported by the overall number of surgeries performed in our region decreasing by $20 \%$, from 304 in 2011 to 247 in 2015 .

The changes noted in this study are important because they change the balance of risks and benefits of prostate cancer screening. Studies from Europe and the United States have also reported an increasing proportion of patients who undergo prostatectomy with intermediate and high-risk disease in recent years. ${ }^{16,17}$ However, these reports have originated from single tertiary care academic centres, therefore changes reported could be a result of referral bias or may only reflect the practice of academic centres. Indeed, a previous report that used data from the large National Cancer Database in the United States, which likely reflects treatment trends in the population, reported that although active surveillance of low-risk disease was increasing, so too was prostatectomy. ${ }^{18} \mathrm{In}$ 2012, 51\% of patients included the National Cancer Database with low-risk disease received surgery.

\section{Strengths and limitations}

We included data from all centres that perform prostatectomy in our region, making the results a nonbiased reflection of trends occurring in our population. Although referral practices may vary, there is no reason to believe that a substantial proportion of patients receive treatment outside this geographical region.

Our relatively long study period compared with previous studies suggests that the changes seen are not transient. Moreover, changes were seen among academic uro-oncologists, community-based urologists, and those performing roboticassisted and open surgical approaches, which suggests that the trends are not a result of referral bias.

Our study does not address the efficacy of surgery. Owing to incomplete preoperative clinical data, it was not possible to calculate the National Comprehensive Cancer Network risk group for all patients; however, given that postoperative pathologic trends were similar, differences in clinical risk are unlikely to be significantly different. It is possible that the trends we saw are partially explained by a greater proportion of patients being diagnosed with clinically significant cancers or a greater proportion of low-risk patients receiving nonsurgical interventions such as radiotherapy. Data to assess these variables were not available for this study. However, a previous report showed that more patients with low-risk disease in our region are opting for active surveillance, which would suggest that patient selection is the main reason for the changes observed. ${ }^{4}$

\section{Conclusion}

This study shows that almost all men who undergo prostatectomy in Eastern Ontario have clinically significant cancer. These data suggest that overtreatment of prostate cancer with surgery is decreasing, which increases the benefits and decreases the risks of prostate cancer screening. Future research should examine treatment trends in other populations and other interventions, such as radiotherapy.

\section{References}

1. Canadian cancer statistics publication. Toronto: Canadian Cancer Society; 2017. Available: www.cancer.ca/en/cancer-information/cancer-101/canadian-cancer -statistics-publication/? region=on (accessed 2018 Feb. 2).

2. Bell N, Gorber SC, Shane A, et al. Recommendations on screening for prostate cancer with the prostate-specific antigen test. CMA7 2014;186:1225-34.

3. Loeb S, Bjurlin MA, Nicholson J, et al. Overdiagnosis and overtreatment of prostate cancer. Eur Urol 2014;65:1046-55.

4. Cristea O, Lavallée LT, Montroy J, et al. Active surveillance in Canadian men with low-grade prostate cancer. CMA7 2016;188:E141-7.

5. Hamdy FC, Donovan JL, Lane JA, et al. 10-year outcomes after monitoring, surgery, or radiotherapy for localized prostate cancer. N Engl 7 Med 2016; $375: 1415-24$.

6. Bill-Axelson A, Holmberg L, Ruutu M, et al. Radical prostatectomy versus watchful waiting in early prostate cancer. N Engl 7 Med 2011;364:1708-17.

7. Thompson I, Thrasher JB, Aus G, et al. Guideline for the management of clinically localized prostate cancer: 2007 update. 7 Urol 2007;177:2106-31.

8. Lavallée LT, Manuel DC, van Walraven C. Survival of men with prostate cancer undergoing radical prostatectomy in Ontario. 7 Urol 2014;192:1385-9.

9. Albertsen PC, Hanley JA, Fine J. 20-year outcomes following conservative management of clinically localized prostate cancer. 7AMA 2005;293:2095-101.

10. D'Amico AV. Biochemical outcome after radical prostatectomy, external beam radiation therapy, or interstitial radiation therapy for clinically localized prostate cancer. $7 A M A 1998 ; 280: 969-74$.

11. Schröder FH, Hugosson J, Roobol MJ, et al. The European randomized study of screening for prostate cancer - prostate cancer mortality at 13 years of follow-up. Lancet 2014;384:2027-35

12. Wilt TJ, Brawer MK, Barhill J, et al. The Prostate Cancer Intervention versu Observation Trial (PIVOT). Oncology 1997;11:1133-9; discussion 1139-40, 1143.

13. Donovan J, Mills N, Smith M, et al. Improving design and conduct of randomised trials by embedding them in qualitative research: Protec $\mathrm{T}$ (prostate testing for cancer and treatment) study. BM7 2002;325:766-70.

14. Klotz L. Active surveillance for prostate cancer: overview and update. Curr Treat Options Oncol 2013;14:97-108.

15. Morash C, Tey R, Agbassi C, et al. Active surveillance for the management of localized prostate cancer: guideline recommendations. Can Urol Assoc 72015 ; 9:171-8.

16. Hayes JH, Ollendorf DA, Pearson SD, et al. Active surveillance compared with initial treatment for men with low-risk prostate cancer: a decision analysis. 7AMA 2010;304:2373-80.

17. Wilcox CB, Gilbourd D, Louie-Johnsun M. Anxiety and health-related quality of life (HRQL) in patients undergoing active surveillance of prostate cancer in an Australian centre. BfU Int 2014;113:64-8.

18. Huland $H$, Graefen $M$. Changing trends in surgical management of prostate cancer: the end of overtreatment? Eur Urol 2015;68:175-8.

Affiliations: Division of Urology (Witherspoon, Lau, Breau, R.N. Morash, Cagiannos, C. Morash, Lavallée), Department of Surgery, The Ottawa Hospital and University of Ottawa; Ottawa Hospital Research Institute (Breau, Knee, Mallick, Lavallée); Division of Obstetrics and Gynecology, Department of Surgery (Fung), The Ottawa Hospital and University of Ottawa, Ottawa, Ont.

Contributors: All of the authors contributed to the concept, design, analysis, writing, or revision of the manuscript. All of the authors approved the final version of the manuscript to the published and agreed to act as guarantors of the results.

Funding: Dr. Lavallée has received research funding support from the Canadian Urological Association Early Investigator Research Scholarship Grant and Prostate Cancer Fight Foundation - Ride for Dad.

Supplemental information: For reviewer comments and the original submission of this manuscript, please see www.cmajopen.ca/content/6/2/ E197/suppl/DC1. 